\title{
Oral Mucositis WHO Grade III
}

National Cancer Institute

\section{Source}

National Cancer Institute. Oral Mucositis WHO Grade III. NCI Thesaurus. Code C124789.

Severe mucositis with oral ulcers; liquid diet only. 